\title{
Observation and Implications of High Mass-to-Charge Ratio Ions from Electrospray Ionization Mass Spectrometry
}

\author{
Brian E. Winger, Karen J. Light-Wahl, Rachel R. Ogorzalek Loo, ${ }^{*}$ \\ Harold R. Udseth, and Richard D. Smith \\ Chemical Methods and Separations Group, Chemical Sciences Department, Pacific Northwest Laboratory, \\ Richland, Washington, USA
}

\begin{abstract}
High mass-to-charge ratio ions ( $>4000$ ) from electrospray ionization (ESI) have been observed for several protcins, including bovine cytochrome $\mathcal{C}\left(M_{\mathrm{r}} 12,231\right)$ and porcinc pepsin $\left(M_{\mathrm{r}} 34,584\right)$, by using a quadrupole mass spectrometer with an $m / z 45,000$ range. The ESI mass spectrum for cytochrome $c$ in an aqueous solution gives a charge state distribution that ranges from $12+$ tn $2+$, with a brnad, low-intensity peak in the mass-to-charge ratio region corresponding to the $[\mathrm{M}+\mathrm{H}]^{+}$ion. The negative ion ESI mass spectrum for pepsin in $1 \%$ acetic acid solution shows a charge state distribution ranging from $7-$ to $2-$. To observe the $[\mathrm{M}-\mathrm{H}]^{-}$ion, harsher desolvation and interface conditions were required. Also observed was the abundant aggregation of the proteins with average charge states substantially lower than observed for their monomeric counterparts. The negative ion ESI mass spectrum for cytochrome $c$ in 1-100 mM NH${ }_{4} \mathrm{OAc}$ solutions showed greater relative abundances for the higher mass-to-charge ratio ions than in acidic solutions, with an $[\mathrm{M}-\mathrm{H}]$ ion relative abundance approximately $50 \%$ that of the most abundant charge state peak. The observation that protein aggregates are formed with charge states comparable to monomeric species (at lower mass-to-charge ratios) suggests that the high mass-to-charge ratio monomers may be formed by the dissociation of aggregate species. The observation of low charge state and aggregate molecular ions concurrently with highly charged species may serve to support a variation of the charged residue model, originally described by Dole and co-workers (Dole, M., et al. J. Chem. Phys. 1968, 49, 2240; Mack, L. L., et al. I. Chem. Phys. 1970, 52, 4977), which involves the Coulombically driven formation of either very highly solvated molecular ions or lower nanometer-diameter droplets. (I Am Soc Mass Spectrom 1993, 4, 536-545)
\end{abstract}

$\mathrm{E}$ lectrospray ionization (ESI) has proved to be an extremely powerful tool for the analysis of large biomolecules $[1,2]$. Owing to the multiple charging characteristic of the ESI process, analysis of large molecules can be performed routinely with conventional quadrupole mass spectrometers. Although a mechanism of macromolecular gas-phase ion formation has yet to be widely accepted, the ion evaporation mechanism proposed by Iribarne and Thomson [3, 4], and more recently supported by results from Kebarle and co-workers [5], is generally believed to be important for small ionic species. Fenn and co-workers [6] have argued that this model offers a better explanation for the production of larger ions than does the

\footnotetext{
*Prescnt address: 2560 MSRB-II, Box 0674, 1150 W. Medical Center Drive, University of Michigan Medical School, Arun Arbor, MI 48109-0674.

Address reprint requests to Richard D. Smith, Chemical Methods and Separations Group, Chemical Sciences Department, Pacific Northwest Laboratory, P.O. Box 999, Mail Stop P8-19, Richland, WA 99352.
}

charged-residue model [7], and they note that "if antecedent droplets contain more than one solute molecule, or a different number of charges, the [Dole] model requires an elaboration"s to explain the resulting mass spectra [6].

The charged-residue model for macromolecular gas-phase ion formation proposed by Dole and coworkers [7] over two decades ago is based on the combination of solvent evaporation and Coulombic explosion(s), resulting in a droplet size sufficiently small such that it contains only one molecular ion (due to retaining some of the original droplet charge). In addition to the concerns noted by Fenn and co-workers [G], a major criticism of the charged-residue model has been that its prediction seems in contrast with experimental observation: Large clusters and other nonvolatile droplet residue should also be observed to be associated with macromolecular ions produced by ESI. In fact, it is possible to obtain protein ESI spectra of proteins that do not suffer from significant "residue" association or solute clustering, and this has been a 
major factor in the success and acceptance of ESI for molecular weight determination of proteins up to $200,000 \mathrm{Da}$. Thus, the field evaporation model continues to be widely cited as the most likely mechanism leading to the formation of even very large molecular ions by ESI.

In opposition to this view, Röllgen and co-workers $[8,9]$ have argued that in the Rayleigh limit, Coulombic explosions would necessarily occur prior to ion evaporation because the electric fields required for this process are locally high [10]. More important, perhaps, they suggest that the droplet deformation that would most likely occur for such a field evaporation process would more likely produce a jet of small charged droplets.

Recently, Nohmi and Fenn [11] studied the ESI of poly(ethylene glycols) (PEGs) with molecular weights up to 5 million and suggested that the ion evaporation mechanism appeared most likely for PEG molecular weights up to approximately $30,000 \mathrm{Da}$, whereas the formation of larger ions could be better attributed to a charged-residue mechanism. Owing to the complexity of the sequence of events most likely contributing and experimental difficulties involved with probing the ESI process, major questions remain concerning the mechanisms of macromolecular ion formation.

The nature of the ESI process is also of interest in that it may ultimately define the constraints on the range of solution interactions that can be probed by ESI mass spectrometry (ESI/MS). The "extreme gentleness" of the ESI ion formation process has been emphasized by the observation that both specific [12] and nonspecific [13] noncovalent associations in solution can be preserved in the gas phase. Nonspecific dimerization has been observed for numerous proteins; however, the maximum charge state observed for dimers is consistently less than twice that of the monomer. For example, the maximum charge state observed for the bovine carbonic anhydrase dimer was $57+$, whereas the maximum charge state for the monomer was $46+$ [13]. For high mass-to-charge ratio (low charge state) macromolecular ions, it might be expected that the reduced Coulombic repulsive forces should favor retention of noncovalent associations for multiply charged species and that specific and nonspecific complexes, aggregates, and perhaps higher order (three-dimensional) macromolecular structures should most likely be preserved in the gas phase. It should also be noted that ESI mass spectra for noncovalent complexes generally contain broad peaks [12] that can often be attributed to adduction by other solution components and impurities. Mass spectrometric interface conditions can greatly influence the qualitative features of ESI mass spectra through the details of heating/activation processes [2]. It appears quite likely that the ESI process initially produces species quite different from those ultimately detected by using mass spectrometry. Insufficient "heating" leads to reduced signal intensities that can be most likely attributed to greater solva- tion or adduction of molecular ions. For example, phosphate, sulfate, and other adducting species can often be removed by collisional heating in the interface [2, 14].

Although earlier ESI/MS studies were restricted to relatively low mass-to-charge ratios, more recent studies have been extended to approximately $m / z 4000$. The absence of higher mass-to-charge ratio ESI studies might perhaps be attributed to wide acceptance of the field evaporation model of ion formation as well as to the limited mass-to-charge ratio range of conventional quadrupole mass spectrometers. Indeed, it has been speculated that ESI might yield "multiple charging so extensive that ion mass-to-charge ratio values are always less than approximately 2500, no matter how massive the parent" [1]. Our interest in high mass-tocharge ratio ESI/MS has been twofold: (1) such a capability may allow the detection of otherwise "Coulombically" labile, noncovalent associations, and (2) investigation of ions from ESI over an extended range provides new insights into the ESI process. In this study, we present our first observations on the formation of high mass-to-charge ratio ions by using an extended mass-to-charge ratio range quadrupole mass spectrometer and discuss issues relevant to high mass-to-charge ratio ion production and speculate on their possible mechanistic implications.

\section{Experimental}

Figure 1 is an illustration of the ESI high-mass quadrupole mass spectrometer constructed in our laboratory, which will be described in detail elsewhere [15]. Briefly, the high-mass quadrupole mass spectrometer was based on a radiofrequency ( $\mathrm{rf}$ ) power supply designed by Extrel Corp. (Pittsburgh, PA) and implemented by Beuhler and Friedman [16]. The fundamental oscillating frequency was $292 \mathrm{kHz}$, compared with 1.2-1.5 $\mathrm{MHz}$ used for the standard Extrel quadrupole

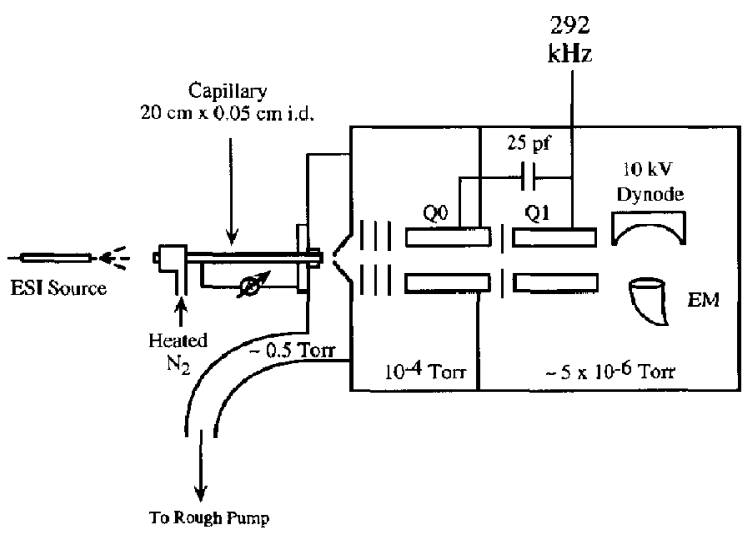

Figure 1. Low-frequency extended mass range quadrupole mass spectrometer used in this study. The vacuum interface consists of a heated metal capillary with a heated countercurrent $N_{2}$ flow to aid in desolvation. $\mathrm{EM}=$ electron multiplier. 
rf power supply. Using bovine cytochrome $c$ (Sigma Chemical Co., St. Louis, MO) as the calibrant and extrapolating from the singly charged molecular ion at $m / z \quad 12,232$, we obtained an experimentally determined maximum of $m / z 45,000$. A standard Galileo 4870 channeltron electron multiplier detector was used in most experiments for detection of ions under approximately $m / z 15,000$. The data for pepsin were obtained by using a $20-\mathrm{kV}$ postacceleration system to enhance the detection efficiency of higher mass-tocharge ratio species. An approximately tenfold increase in sensitivity at high mass-to-charge ratio was observed with this detector relative to that of the standard detector.

Interestingly, an unexpected problem arose with the use of high-dynode voltages. At low if potentials applied to the quadrupole, very large (and perhaps highly solvated) charged particles of high mass-to-charge ratio, having high kinetic energies (voltages in excess of $400 \mathrm{~V}$ were necessary to prevent their detection) and most likely arising owing to the expansion into vacuum, traversed the rf and $\mathrm{rf} / \mathrm{dc}$ mass filters and impinged on the detector. This caused intense noise spikes at higher detector anode voltages, with frequencies of 10 counts/s to several thousand counts per second. These events, which were not mass analyzed, interfered with mass detection below $m / z 2500$ and prevented the observation of higher charge state ions unless the dynode voltage was reduced during the early part of a scan.

Data were acquired by using a Vector One (Teknivent, Inc., St. Louis, MO) data system. The mass-tocharge ratio limit for the quadrupole version of the Vector One is 9999; therefore the upper mass-to-charge ratio limit of the high-mass quadrupole mass spectrometer, $m / z 45,000$, was entered into Vector One as 9000. This limited the smallest mass-to-charge ratio step size to 5. The typical scan rate was 121 (mass-tocharge ratio) $/ \mathrm{s}$. Thus, a spectrum ranging from $\mathrm{m} / \mathrm{z}$ 1000 to 45,000 took approximately $6 \mathrm{~min}$ to acquire. Data presented here represent the average of five scans over the mass-to-charge ratio range of interest.

The electrospray interface consisted of a resistively heated stainless steel capillary $(1.59-\mathrm{mm}$ nominal o.d., $0.5-\mathrm{mm}$ i.d., $20 \mathrm{~cm}$ long), similar to the design of Chait and co-workers [17], with an auxiliary assembly positioned at the capillary entrance to deliver heated countercurrent $\mathrm{N}_{2}$ gas to assist ion desolvation. Capillary heating is reported as watts applied across the capillary in addition to the approximate temperature as measured on the external surface of the capillary. In addition, the voltage difference applied between the capillary and skimmer $(\Delta C S)$ is also given. Proton transfer reactions with diethylamine were performed on the high-mass quadrupole mass spectrometer with a "Y-tube" reaction inlet, as described elsewhere [18]. The electrospray source implemented in these studies was based on a coaxial sheath design developed by Smith et al. [19]. Unless otherwise noted, sample flow rates were between 0.5 and $1.0 \mu \mathrm{L} / \mathrm{min}$, and the methanol sheath flow was $3.0 \mu \mathrm{L} / \mathrm{min}$. An additional coaxial yas flow of sulfur hexafluoride was also used to stabilize the formation of electrosprayed ions by diminishing the probability of corona discharge.

All samples used in this study were oblained from Sigma and were used without further purification. Sample concentrations for each analyte were $1 \mu \mathrm{g} / \mu \mathrm{L}$, except where noted. Bovine cytochrome $c\left(M_{\mathrm{r}} 12,231\right)$ was examined in doubly distilled deionized water $(\mathrm{pH}$ $\sim 7.5$ ) and in $1-100 \mathrm{mM}$ aqueous $\mathrm{NH}_{4} \mathrm{OAc}$ solutions. In an attempt to alter the observed charge state distribution of a protein to higher mass-to-charge ratio, porcine pepsin, a highly acidic protein $\left(M_{\mathrm{r}} 34,584\right)$, was dissolved in $5 \%$ acetic acid/distilled deionized water and examined in the negative ion ESI mode.

\section{Results}

Figure 2 shows the mass spectra for three different proteins: (1) bovine ubiquitin in an aqueous $5 \%$ acetic acid solution, (2) bovine cytochrome $c$, and (3) equine myoglobin in denaturing 47.5:47.5:5 $(\mathrm{v} / \mathrm{v} / \mathrm{v})$ $\mathrm{MeOH} / \mathrm{H}_{2} \mathrm{O} / \mathrm{HOAc}$ solutions. Several features are evident in these spectra. The charge state distributions appear to be shifted to higher mass-to-charge ratio compared with standard quadrupole mass spectrometers. With the heated capillary inlet, the extent of proton transfer reactions with small solvent molecules can serve to reduce the extent of multiple charging for the gas-phase protein. In addition, experiments performed with the same instrumental configuration, but by using a high-frequency rf power supply $(1.3 \mathrm{MHz})$, yielded a charge state distribution ranging from $18+$ to $10+$ for bovine cytochrome $c$. This suggests that the major contributor to the observed shift in the charge state distribution is discrimination against low massto-charge ratio species with the low-frequency quadrupole.

It is also evident from Figure 2 that the extent of protein aggregation observed is much greater than had been observed previously at lower mass-to-charge ratios, especially in the case of ubiquitin [13]. Higher order aggregation has also been observed previously in ESI/MS for small amino acids such as arginine [20].

Finally ${ }_{r}$ Figure 2 demonstrates that ions of relatively high mass-to-charge ratio can be produced by ESI. The charge state distributions observed in ESI mass spectra for proteins have been shown to be somewhat dependent on the solution composition, which can affect conformation of the protein $[2,21-23]$. The compact globular structure, often characteristic of native proteins, can be disrupted by drastic $\mathrm{pH}$ changes, organic solvents, strong denaturing agents (such as urea), and increased temperature [24]. As an example, the positive ion FST mass spectrum for cytochrome $c$ at $\mathrm{pH} 2.6$ results in a charge state distribution of ions that ranges from $19+$ to $11+[22]$. By increasing the $\mathrm{pH}$ to 3.8 , a bimodal charge state distribution was observed. The 

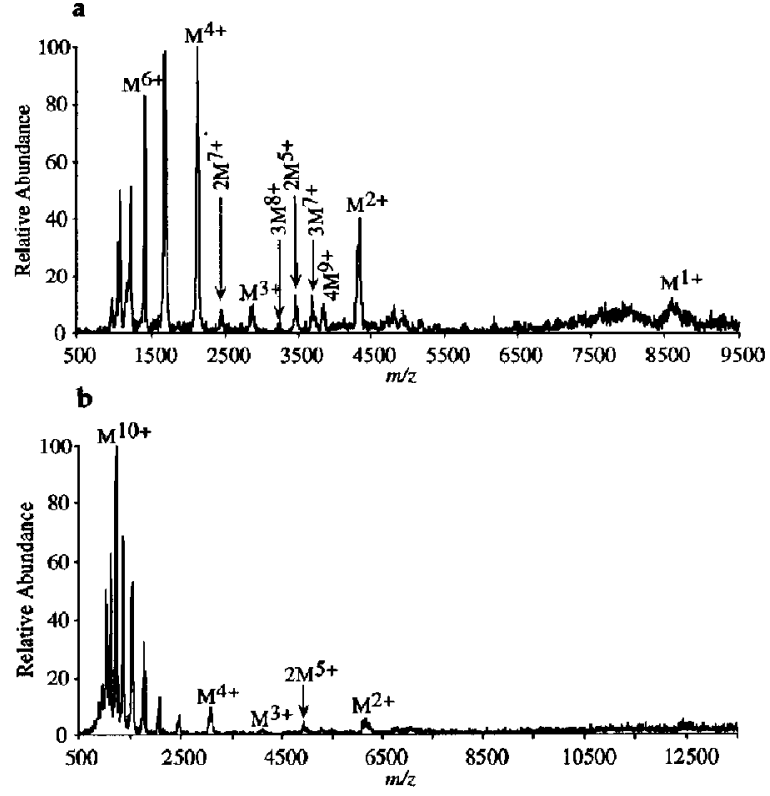

c

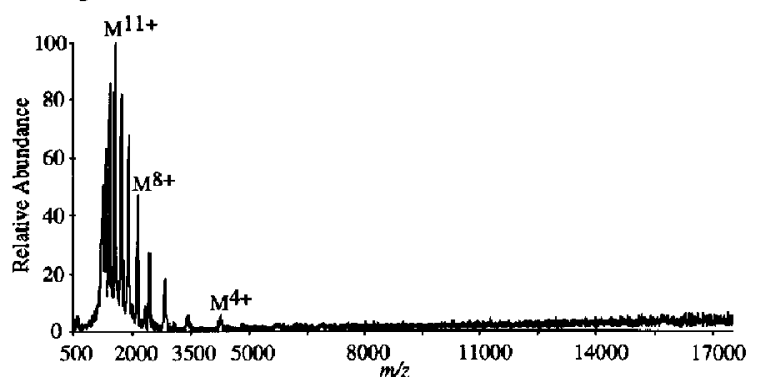

Figure 2. Examples of the extended mass-to-charge ratio range positive ion EST mass spectra obtained for (a) bovine ubiquitin, (b) bovine cytochrome $c$, and (c) equine myoglobin. The solution and interface conditions used to obtain these data are as follows: (a) aqueous $5 \% \mathrm{HOAc}$, inlet capillary heated by $31 \mathrm{~W}\left(\sim 200^{\circ} \mathrm{C}\right)$, $\Delta \mathrm{CS}=75 \mathrm{~V}$; (b) $50: 50 \mathrm{MeOH} / \mathrm{H}_{2} \mathrm{O} 5 \% \mathrm{HOAc}$, inlet capillary heated by $31 \mathrm{~W}, \Delta \mathrm{CS}=90 \mathrm{~V}$; and (c) $50: 50 \mathrm{MeOH} / \mathrm{H}_{2} \mathrm{O} 5 \%$ HOAc, inlet capillary heated by $31 \mathrm{~W}, \Delta \mathrm{CS}=100 \mathrm{~V}$. (Monomer species are labeled $M^{4+}$, etc., which denotes $[M+4 H]^{4+}$; similarly, dimer ions are labeled $2 \mathrm{M}^{7+}$, etc.)

higher charge state distribution ranged from $17+$ to $12+$, whereas the lower charge state distribution ranged from $10+$ to $7+$ (where the $7+$ ion was the highest mass-to-charge ratio species observable with the mass spectrometer utilized). Attempts have been made to correlate such observations with protein charge state in solution [21, 23, 25]. Based on such observations, a solution $\mathrm{pH}$ closer to neutrality might be expected to yield lower ESI charge states. Figure 3 shows the ESI mass spectrum of bovine cytochrome $c$ in doubly distilled deionized $\mathrm{H}_{2} \mathrm{O}$. The monomer charge state distribution ranges from $12+$ to $2+$, with the $[\mathrm{M}+8 \mathrm{H}]^{8+}$ peak the most abundant. Protein aggregates are also observed between $m / z 2000$ and

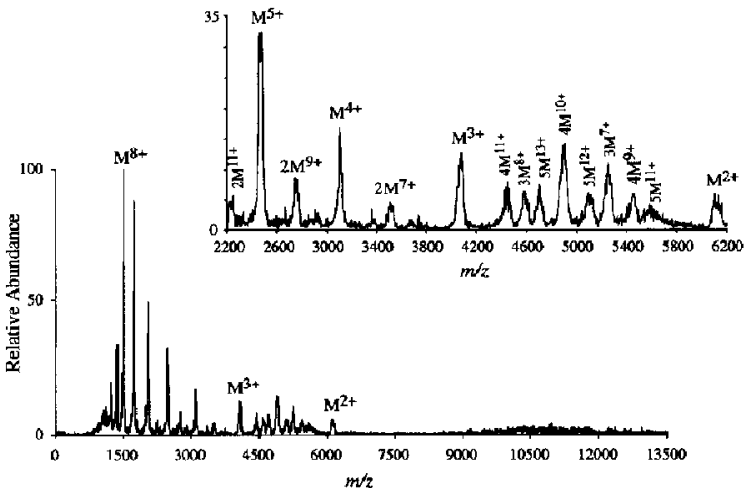

Figure 3. Positive ion ESI mass spectrum of bovine cy tachrome $c$ in doubly distilled deionized $\mathrm{H}_{2} \mathrm{O}\left(1 \mu \mathrm{g} / \mu \mathrm{L}=8 \times 10^{-5} \mathrm{M}\right)$. Inset: detail of the portion of the spectrum where multimers are observed. The multiply protonated monomer ions are observed from $[\mathrm{M}+12 \mathrm{H}]^{12+}$ to $[\mathrm{M}+2 \mathrm{H}]^{2+}$. In addition, peaks corresponding to cytochrome $c$ dimers (2M), trimers (3M), tetramers (4M), and pentamers (5M) are observed between $m / z 2000$ and 6000 with the charge states indicated. The inlet capillary was heated by $30 \mathrm{~W}\left(\sim 195^{\circ} \mathrm{C}\right) ; \Delta \mathrm{CS}=100 \mathrm{~V}$. It should be noted that several of the aggregate peaks could have more than one assignment (i.e., $m / z \quad 6117=\mathrm{M}^{2+}, 2 \mathrm{M}^{4+}, 3 \mathrm{M}^{5+}$, etc.), whereas other mass-to-charge ratio values have only one reasonable assignment (i.e., $m / z \quad 5240=3 \mathrm{M}^{7+}$ ). The relative abundances of these "unique" ions were used to estimate the contributions of related ions to the unknown peaks.

6000 corresponding to the following degrees of aggregation and charge state distributions: dimers $11+$ to $5+$; trimers $8+$ and $7+$; tetramers $11+$ to $9+$; pentamers $13+$ to $11+$. Varying concentrations of cytochrome $c$ in $\mathrm{H}_{2} \mathrm{O}$ from 0.1 to $10 \mu \mathrm{g} / \mu \mathrm{L}$ were analyzed, with less than a factor of 3 increase in the relative extent of aggregation observed.

In contrast to the extensive aggregation observed for cytochrome $c$ in pure $\mathrm{H}_{2} \mathrm{O}$, EST/MS of a $10-\mathrm{mM}$ $\mathrm{NH}_{4} \mathrm{OAc}$ aqueous solution of cytochrome $c$ (Figure 4) yielded a greatly reduced extent of aggregation; however, the lower mass-to-charge ratio (higher charge state) monomer ion distribution was similar. The increased electrolyte concentration apparently reduces the protein-protein interactions in solution, lowering the extent of aggregation observed by ESI/MS. Varying the concentration of $\mathrm{NH}_{4} \mathrm{OAc}$ from 1 to $100 \mathrm{mM}$ did not significantly alter the observed charge state distribution or the extent of aggregation of cytochrome $c$ observed by ESI/MS.

The high $\mathrm{NH}_{4} \mathrm{OAc}$ concentration would necessarily result in an increase in the $\mathrm{NH}_{3}(\mathrm{~g})$ concentration during solvent evaporation (relative to lower $\mathrm{NH}_{4} \mathrm{OAc}$ concentratiuns), and the possibility of lowering the charge state of multiply charged proteins by proton transfer reactions should be considered. Previous results obtained in this laboratory [18] and elsewhere [26] indicated that proton transfer reactions between gas-phase bases such as $\mathrm{NH}_{3}$ and multiply protonated proteins result in a shift in the charge state distribu- 


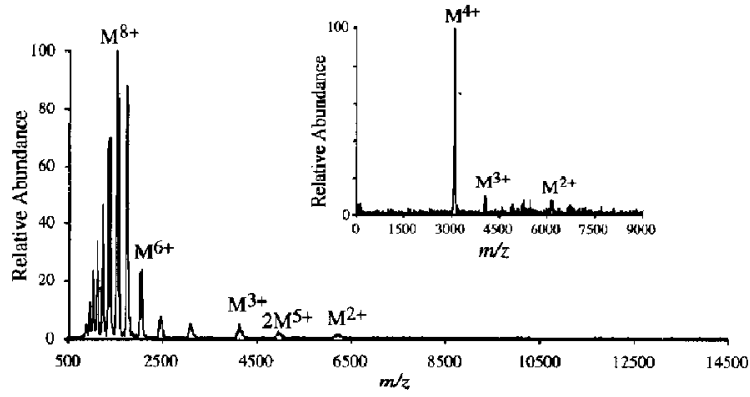

Figure 4. Positive ion EST mass spectrum of bovine cytochrome c $(1 \mu \mathrm{g} / \mu \mathrm{L})$ in aqueous $10 \mathrm{mM} \mathrm{NH}{ }_{4} \mathrm{OAc}(\mathrm{pH} 6.7)$. Monomer species domirate with charge states from $13+$ to $2+$. The inlet capillary was heated by $29 \mathrm{~W}\left(\sim 190^{\circ} \mathrm{C}\right) ; \Delta \mathrm{CS}=70 \mathrm{~V}$. Inset: positive ion ESI mass spectrum of bovine cytochrome $c$ in doubly distilled deionized $\mathrm{H}_{2} \mathrm{O}$ after reactions with triethylamine in the inlet "Y-tube" reaction chamber [18]. The charge state distribution has been significantly shifted to the range $4+$ to $2+$. Additional triethylamine does not shift the charge state distribution to lower charge states. The irlet capillary was heated by 32 $\mathrm{W}\left(\sim 205^{\circ} \mathrm{C}\right) ; \Delta \mathrm{CS}=100 \mathrm{~V}$

tion. These proton transfer reactions continue until the reactivity of the remaining charge states is negligible or until the charge state distribution is beyond the mass-to-charge ratio range of the instrumentation. These reactions involved much greater $\mathrm{NH}_{3}$ gas-phase concentrations than could be realized by the $\mathrm{NH}_{1} \mathrm{OAc}$ concentrations used in the present case. The gas-phase reaction between aqueous cytochrome $c$ and triethylamine was monitored to completion (as defined above). The partial pressure of triethylamine was increased to the point where an additional increase did not cause any further change in the charge state distribution. Under these conditions, the $[\mathrm{M}+4 \mathrm{H}]^{4+}$ species was the highest charge state observed and was also the base peak (Figure 4, inset). Most notably, the same lower charge states that were observed in Figures 3 and 4 (i.e., $4+$ to $2+$ ) were also observed when triethylamine was added. It appears that higher charge states can undergo proton transfer with triethylamine but that the $[\mathrm{M}+4 \mathrm{H}]^{4+}$ species has protonation sites that have effective proton affinities [18] too high to permit additional reactions with triethylamine. These results suggest that although the formation of lower charge state ions via proton transfer in the interface is feasible, this is not the major contributor to low charge state ion formation here or under typical ESI conditions. Nevertheless, reactions as demonstrated here may be advantageous for applications that benefit either from reduced molecular ion charging or by narrowing the distribution of charge states.

Figure 5 gives the negative ion ESI mass spectrum of cytochrome $c$ in $10 \mathrm{mM} \mathrm{NH}_{4} \mathrm{OAc}(\mathrm{pH} \mathrm{6.7)}$, showing an increased extent of aggregation and increased abundances of high mass-to-charge ratio ions, which is in sharp contrast to the corresponding positive ion charge state distribution (Figure 4). The peaks corresponding

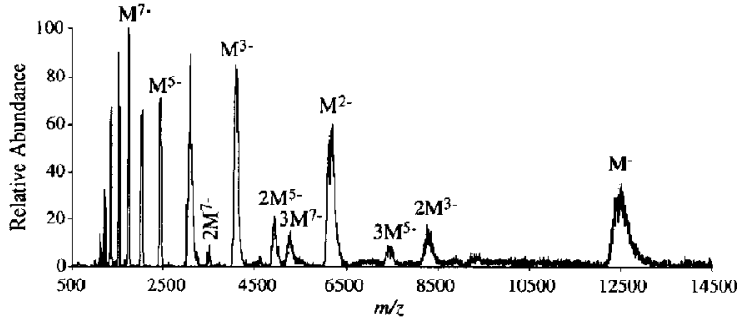

Figure 5. Negative ion ESI mass spectrum of bovine $\mathrm{cy}^{\text {- }}$ tochrome $c(1 \mu \mathrm{g} / \mu \mathrm{L})$ in aqueous $10 \mathrm{mM} \mathrm{NH}_{4} \mathrm{OAc}$. The charge state distribution of the monomer ions ranges from $12-$ to $1-$. Dimers and trimers are also observed in greater abundance than were observed for the same solution in the positive ion mode (see Figure 4). The inlet capillary was heated by $29 \mathrm{~W}\left(\approx 190^{\circ} \mathrm{C}\right)_{r}$; $\Delta \mathrm{CS}=70 \mathrm{~V}$.

to the 4 - to 2 - monomer charge states at $m / z 3059$, 4078 , and 6117 almost certainly have additional contributions from dimer and trimer species that are observed at the same mass-to-charge ratio as the monomer ions. The most likely assignments for the other ions observed are $m / z 3490,[2 \mathrm{M}-7 \mathrm{H}]^{7 \cdots} ; m / z$ 4585, $[3 \mathrm{M}-8 \mathrm{H}]^{8-} ; m / z$ 4890, [2M $\left.-5 \mathrm{H}\right]^{5-} ; m / z$ 5240, $[3 \mathrm{M}-7 \mathrm{H}]^{7-} ; m / z$ 7337, [3M $\left.-5 \mathrm{H}\right]^{5-;} m / z$ 8153, $[2 \mathrm{M}-3 \mathrm{H}]^{3-}$; and $m / z$ 9172, $[3 \mathrm{M}-4 \mathrm{H}]^{4-}$. Increasing the temperature of the inlet capillary was found to result in a decrease in those ions assumed to be aggregates and a moderate decrease in the intensity of the peaks dominated by the $[\mathrm{M}-4 \mathrm{H}]^{4-},[\mathrm{M}-$ $3 \mathrm{H}]^{3-}$, and $[\mathrm{M}-2 \mathrm{H}]^{2-}$ species. We attribute this apparent loss of low charge state monomers with increasing interface heating to decreases in the aggregate components that appear at the same mass-to-charge ratio as these assigned monomer ions. Aggregates are more labile than monomer species, and peaks attributed to the former decrease in relative abundance as the capillary temperature is increased.

Altering the solution and spray conditions for ESI can result in the observation of different charge state distributions, as illustrated in the previous examples. Porcine pepsin $\left(M_{r} 34,584\right)$ is a highly acidic, 326residue protein [27] with 29 Asp and 13 Glu residues. In an attempt to observe high mass-to-charge ratio (low charge state) negative ions, the ESI negative ion mass spectrum for pepsin dissolved in $1 \%$ acetic acid ( $\mathrm{pH}-2.5$ ) was obtained (Figure 6a). Approximately $38 \mathrm{~W}$ was applied to the inlet capillary, with a countercurrent gas flow at ambient temperature. The charge state distribution ranges from $7-$ to $2-$, substantially lower than that observed for basic solutions where the maximum charge state was $42-$ [2]. Peaks corresponding to pepsin aggregation were also observed, with contributions evident for dimers and (to a lesser extent) trimers. Another interesting feature is the large unresolved distribution from $m / z 25,000$ to 40,000 and a second broad, not readily assignable distribution at approximately $\mathrm{m} / \mathrm{z} 16,000$ (i.e., about half that of the first). The possible origins of these features include 

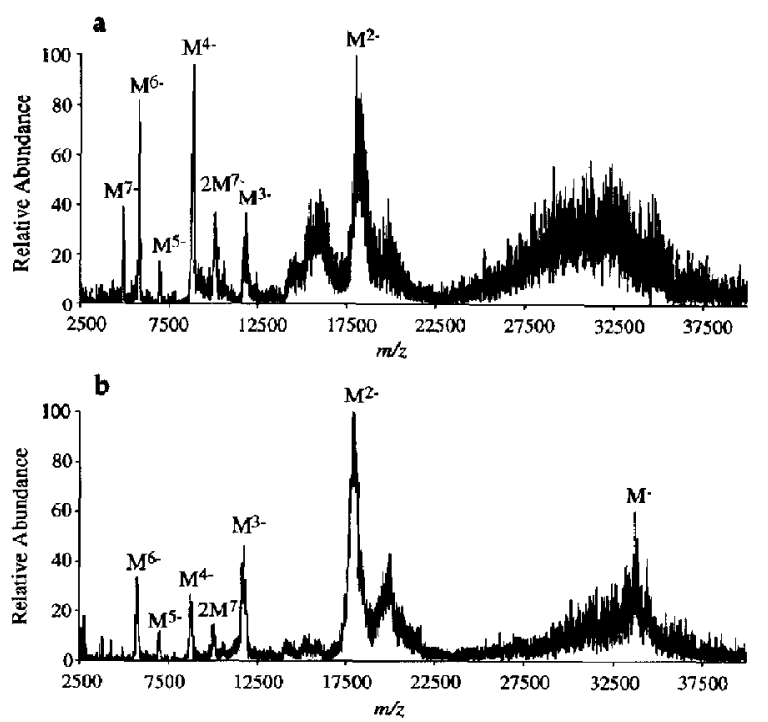

Figure 6. Negative ion ESI mass spectra of bovine pepsin in $5 \%$ HOAc with (a) room temperature countercurrent $\mathrm{N}_{2}$, and (b) heated countercurrent $\mathrm{N}_{2}\left(\sim 80^{\circ} \mathrm{C}\right)$ to aid in desolvation of the higher mass-to-charge ratio ions. The inlet capillary was heated by $38 \mathrm{~W}\left(\sim 240^{\circ} \mathrm{C}\right) ; \Delta \mathrm{CS}=100 \mathrm{~V}$ for both spectra.

molecular ion dissociation products, higher order aggregation, and incomplete desolvation. Collisional and thermal activation studies performed at the ESI interface suggest that these peaks are not due to dissociation products because they do not increase in relative abundance as temperature is increased.

Increasing the temperature of the countercurrent $\mathrm{N}_{2}$ gas flow to $80^{\circ} \mathrm{C}$ reduces some of these broad, unresolved peaks, as shown in Figure $6 \mathrm{~b}$, and a more distinct peak is observed at $m / z 33,750$, which corresponds to the $[\mathrm{M}-\mathrm{H}]^{-}$ion within experimental uncertainty. (The exact mass-to-charge ratio of this and other species at higher mass-to-charge ratios is uncertain owing to the lack of an appropriate calibration compound and to the necessity of extrapolating the calibration from lower mass-to-charge ratios.) We also observe that the relative abundances of the pepsin aggregates decrease under these conditions. The dramatic effect of increasing the countercurrent gas temperature most likely arises because there is a relatively ineffective desolvation of higher mass-to-charge ratio ions by collisional excitation in the interface (due to the low center-of-mass collision energies), which plays a large role in desolvation at low mass-to-charge ratios [2]. In this high mass-to-charge ratio region, the use of a heated countercurrent dry gas flow can remove excess solvent, and desolvation can occur to a greater extent in the heated capillary. The greater extent of desolvation may allow subsequent heating in the capillary (perhaps assisted by any residual effect of collisional activation in the capillary/skimmer region) to increase the effective temperature of the aggregates and enhance their dissociation rates.

\section{Discussion}

The limited mass-to-charge ratio range of mass spectrometers typically used with ESI (i.e., quadrupoles) has largely precluded the observation of low charge state, high mass-to-charge ratio species. Their observation in this work suggests that it is appropriate to reconsider the models proposed to explain the ion formation processes occurring in ESI. It is our purpose in the remainder of this report to discuss the possible origins of our observations and to attempt to qualitatively place them in the context of an admittedly speculative model. We do so knowing that we step beyond the bounds of what is firmly established. We do this for the purpose of elaborating a somewhat different view of the ESI process for macromolecules and in the hope that we will stimulate further experimentation and discussion on questions that, to us at least, appear unresolved.

Dole and co-workers' [7] initial electrospray studies involved the study of polystyrene with molecular weights of up to 860,000 and led to their suggestion that low charge state macroions were produced by ESI. It was proposed that gas-phase macroion formation was a result of solvent evaporation from the initially formed droplet population, yielding one macroion per droplet (although the polymer concentrations used by Dole and co-workers [7] corresponded to more than one molecule per parent droplet on the basis of the droplet size now known to be produced). Nohmi and Fenn [11] have also suggested that a charged-residue model most likely holds for the specific case of large PEG oligomers but argue that the ion evaporation model provides a more coherent explanation for other experimental observations [6]. Among the experimental evidence cited [6] in support of an ion evaporation process are the following: (1) aggregates of arginine are formed under one set of solution conditions but not another at the same solution concentration; (2) a broad distribution of charge states is typically observed for macromolecules by ESI/MS; (3) low-molecular-weight species $(<800)$ are generally observed to be singly charged by ESI/MS; (4) different molecules of the same size give different extents of charging; and (5) different biopolymers give different charge state distributions, and larger ions can be more highly charged than smaller ions. As noted by Fenn and co-workers [6], such results are difficult to rationalize by a simple charged-residue model in which ions arise directly from the parent droplet population (after combinations of solvent, charge, and expelled small droplets are lost).

Two significantly different forms of the chargedresidue model are now considered. The first, and most widely invoked, is that noted above, which considers the residue that arises to be due to solvent evaporation, in conjunction with Coulombically assisted loss of solvent, charge, and the formation of much smaller droplets from the parent droplets. We believe, how- 
ever, that a variation on the charged-residue model, similar to that suggested by Lüttgens and Röllgen [28], offers a more reasonable explanation of experimental observations for macromolecular ion formation, including those reported here. This variation is concerned primarily with the smaller "offspring" droplets (or perhaps their progeny) from which molecular ions are finally produced. The much greater number of offspring druplets, their lower mass-to-charge ratios, and the smaller amount of "residue" that would result suggest to us that this variation of the charged-residue model is more likely to be relevant to ESI/MS. This is the variation that we implicitly refer to in the remainder of this report.

If this variation of the charged-residue model is viable, then one would expect to observe macromolecular ions that contain particle residue under mild ESI interface conditions, the extent of which would be related to the precursor droplet size. With increasingly harsh ESI/MS interface conditions, however, the amount of "particle residue" might decrease and, depending on its nature, eventually result in the formation of a bare macroion. In fact, this is qualitatively the trend observed for small to moderately sized proteins.

Additional information can be obtained from lowresolution ion mobility experiments at atmospheric pressure. An ion mobility analysis of cytochrome $c$ in $50: 50(\mathrm{v} / \mathrm{v})$ water/methanol with $2 \%$ acetic acid [2] yielded an ion mobility spectrum that contained three peaks. The first peak was related to the solvent, the second peak arose from the protein, and the third was ascribed to residual particles (containing protein) that had large mass-to-charge ratios. The intensity of this residue peak became significant only at higher protein concentrations.

With mild interface conditions, large, unresolved ion distributions at high mass-to-charge ratios are observed with ESI/MS with the extended mass-to-charge ratio instrument. This is shown in Figure $6 \mathrm{a}$, where the effectively mild interface conditions resulted in unresolved distributions beyond $m / z$ 13,000, whereas at the same time, higher charge state ions corresponding to pepsin were observed. Increasing the temperature of the countercurrent $N_{2}$ gas (Figure $6 \mathrm{~b}$ ) resulted in increased desolvation, which was observed as increased absolute abundances of the pepsin molecular ions. Most notably, an ion population at approximately $m / z$ 33,500 , in the vicinity of the singly charged molecular anion, was observed. Therefore, the unresolved distributions may be a result of molecular ions, or their aggregates, and represent the charged residue of the smaller "offspring" droplets, not the larger parent droplets. The detected count rates for these higher mass-to-charge ratio species are far too large, and their mass-to-charge ratios too low, for them to arise directly from the original parent particles.

As we described earlier (see Experimental), at low rf levels and high dynode voltages $(>10 \mathrm{kV})$, we ob- served a background signal of up to about several thousand counts per second and not mass-to-charge ratio resolved. We attribute this signal to large residual particles formed from the parent droplets. The presumed very large mass-to-charge ratios of these particles, posisibly grealer than $10^{5}$, would require relatively high rf levels before their trajectories through the quadrupole mass filter would be perturbed. Consistent with this hypothesis is the observation that their signal is reduced (but not completely eliminated) by using countercurrent gas flow. In addition, this conjecture is not inconsistent with the small amount of more direct evidence that exists with regard to ESI droplet size. Direct measurements of the residual particle size have indicated that in addition to one population of larger particles arising from the micron-sized parent droplets, there is a second much smaller distribution ( $<0.1-\mu \mathrm{m}$ initial droplet diameter), the size of which apparently increases with solute concentration [29]. Assuming that these progeny of the parent droplets are the precursors of the observed molecular ions, we find it unclear whether subsequent droplet breakup occurs (as frequently postulated) or whether the initial offspring droplet size will be small enough to be consistent with existing data. (With no firm basis for doing so, we guess that an initial offspring droplet size of $<10 \mathrm{~nm}$ before evaporation would be necessary.)

It might be argued that a field evaporation process would produce only low mass-to-charge ratio ions, and this expectation is often implicit in discussions of this mechanism. This model, in its simplest form, projects that a large, highly charged molecular ion would "lift off" the droplet surface, most likely in a somewhat solvated state, in a manner directly analogous to that proposed for small ions [3, 4]. There are some obvious questions that arise with this model. Would a large molecular ion desorb by "lifting off" at all charge sites? Why are structurally different forms of the same protein (i.e., native versus denatured versus disulfide reduced) found to be ionized with approximately equal efficiencies [2]? Although Fenn and co-workers [6] argue an opposing view, this lack of dependence on structural detail seems to conflict with a field evaporation process. Fenn and co-workers [6] suggest that the dependence of a detected charge state on structural differences can be attributed to differences in molecular properties, such as hydrophobicity. We believe that it is also possible to account for these differences by other factors, including molecular structure, the nature of charge sites and their location, and (perhaps most important) the competition for charge in both solution and gas-phase ion-molecule processes during ESI.

Neither of the models under consideration invokes a direct relationship between ion charge state in solution and that detected by ESI/MS. In addition to the questions raised by high mass-to-charge ratio ion production is the observation that molecular ion charge 
state in solution also appears to be of only indirect importance in ESI. Although we first noted a correlaLion between the extent of charging in positive ion ESI with the number of likely protonation sites $[21,25]$, it is apparent that such a correlation is only rough and that numerous exceptions exist. It is clear that the ion-formation process is not simply the transfer of an ion from solution into the gas phase, but that the overall ESI process itself must play an important role in determining the resulting charge state distribution. It has previously been observed that both positive and negative multiply charged molecular ions can be produced from the same solution [30], depending on ESI polarity. This ESI characteristic is also illustrated by Figures 4 and 5 for cytochrome $c$. (It is also evident that the degree of aggregation depends not only on solution conditions but ESI polarity as well.) Thus, a protein molecular ion in solution can have its net charge and polarity modified by the ESI process, with the resulting maximum charge state seemingly correlated with the number of readily available charge sites having the polarity determined by the ESI process. In our opinion, the fact that ionization occurs with roughly comparable efficiency for both positive and negative ions from the same solution seems to conflict with an ion evaporation process. Even here, however, ambiguity remains because the conditions at the surface of an electrosprayed droplet might alter the net protein charge and perhaps serve to propel an ion evaporation process. Similarly, droplet charge may alter solution conditions sufficiently to either enhance or decrease aggregation.

An attraction of the modified charged-residue model is that it provides a framework for explaining a wide range of observations. First, it rationalizes both the gentle nature of the ESI process and the fact that relatively weak noncovalent complexes can be transferred to the gas phase, such as structures that might be more likely to dissociate under the field stress of an ion evaporation process. Second, a small nanodroplet size for the parent droplet offspring (or their subsequent progeny) explains the lack of extensive droplet residue, which would be primarily concentrated in the large-residue particles from the "parent" droplet. The small amount of residue from such nanodroplets might be effectively removed by heating in the ESI interface, which would be qualitatively consistent with previous observations [2, 14]. Finally, polyelectrolyte charge in solution would be expected to be relatively unimportant in determining the gas-phase charge state. The gas-phase charge state would be determined by droplet charge, the competition for charge retention between potential polyelectrolyte charge sites and other solution components, and (finally) gas-phase processes. For example, the good correlation between maximum ESI positive ion charge state and the number of basic residues may simply reflect the reasonable situation where lower proton affinity charge sites do not com- pete effectively for charge. We previously showed that the observed charge state distribution is subject to significant modification, owing to the effect of Coulombic forces on gas-phase processes [2, 18, 31].

It is our opinion that attempts to distinguish the ion evaporation model from the present variation of the charged-residue model will be extremely difficult. One mechanism involves the formation of progeny droplets that may not be much larger than the molecular ion, whereas the other envisions field evaporation of a substantially solvated molecular ion. As we suggested in an earlier review [25], the products of these processes may be indistinguishable. Boyd and co-workers [32] recently made the same suggestion on the basis of their studies of smaller cluster ions. The present results do suggest one difference that may be subject to experimental verification: Dissociation of aggregates in low charge states would very likely also form neutral monomer species that could not form by ion evaporation. Detection of these products, however, requires reionization and will be extremely difficult because their concentrations are low.

One can ask about the initial size of the progeny droplets in this charged-residue model, but no substantial basis yet exists for $j$ ts determination. Inspection of the mass spectra from this work, however, suggests one interesting possibility. A consistent feature of our observations is that the most abundant aggregate species have average monomer subunit charge states that are substantially lower than those of the free monomer species. If the aggregate species were charging to the same extent as the monomer species, then they should be observed over the same mass-to-charge ratio range. For example, the highest average charge state of a cytochrome $c$ molecule contained in a trimer aggregate is approximately $3+$, whereas the highest observed monomer ion charge state is $10+$ in the same spectrum. The lower average charge state observed for molecules in such noncovalently bound aggregate species might be due to the increased stability of aggregates (where the average charge state is low and Coulombic repulsive forces are not important). On the other hand, there is insufficient evidence on which to assume that higher charge state clusters must be unstable. In particular, it should be noted that cytochrome $c_{s}$ carbonic anhydrase, and other proteins can produce noncovalent dimers detectable by ESI/MS having $m / z<1000$ [13]. Nevertheless, the similar average charge observed for both monomer and aggregate molecular ions suggests the possibility that the precursor droplet size (or at least its charge) might be independent of molecule (or aggregate) size. Alternatively, the similarity of charge states for monomers and aggregates observed here may simply be fortuitous, or molecular size might even be a factor in determining the initial nanodroplet size.

We can also speculate that the aggregate species play a role in the production of the high mass-to-charge 
ratio molecular ions observed in this work. In cases where we have observed molecular ions at greater than approximately $m / z 6000$, aggregate ions are also observed in the spectra (or are observed at gentler interface conditions), with substantial relative abundance. This suggests that high mass-to-charge ratio ions can result from a cascade of aggregate dissociation steps. For example, a trimer with seven charges might dissociate as follows:

$$
[3 \mathrm{M}+7 \mathrm{H}]^{7+} \rightarrow[2 \mathrm{M}+4 \mathrm{H}]^{4+}+[\mathrm{M}+3 \mathrm{H}]^{3+}
$$

followed by

$$
[2 \mathrm{M}+4 \mathrm{H}]^{4+} \rightarrow[\mathrm{M}+\mathrm{H}]^{+}+[\mathrm{M}+3 \mathrm{H}]^{3+}
$$

In conjunction with other charge-loss mechanisms, such aggregate dissociation may be significant in the present study. We note that Lüttgens and Röllgen [28] postulated that aggregates, as precursors to the low mass-to-charge ratio molecular ions, might be observed in ESI/MS.

The significant contributions of ESI interface conditions and the processes that occur after droplet formation substantially determine the species ultimately observed in ESI/MS. Aggregate species do form, at least under some ESI conditions, and probably play a role in determining the charge state distributions observed at higher solute concentrations (i.e., the shift to lower charge state [25]). This shift may be due to both the formation of aggregates and their subsequent dissociation in the interface. The dissociation of a single dimer charge state of carbonic anhydrase was shown previously [13] to produce a distribution of charge states that is almost as broad as the initial monomer charge state distribution. Therefore, it is possible that the dissociation of the small "particles" produced by a charged-residue process would yield an analogous charge state distribution, giving rise to the charge state distributions observed in ESI/MS, not strongly dependent on the initial distribution of nanodroplet charge and size.

Additional experimental studies may serve to further clarify details of the ESI process. For example, studies over a range of solutes, solution conditions, and concentrations would be informative. Our limited study of concentration covered two orders of magnitude but showed only a limited change in the amount of aggregation. Ion mobility studies suggest the possibility that most of the additional solute contributes to the large "residue particles" [2], but alternative interpretations are possible. Clearly, the simple models advanced to date cannot fully account for the range of observations available, or, at the very least, they need to be augmented by a better understanding of the detailed chemical and physical phenomena.

Finally, a reconsideration of the early ion mobility results of Dole and co-workers [33] may be appropriate. Lysozyme in 90:10 $(\mathrm{v} / \mathrm{v})$ ethanol/water solution was reported to yield three peaks that were qualitatively similar to those from ion mobility studies done in this laboratory [2]. Dole and co-workers [33], however, suggested a lower extent of charging (and higher mass-to-charge ratio) than typically observed by ESI/MS. They attributed three separate peaks to the singly and doubly charged monomers and the triply charged trimer. Our studies with cytochrome $c$ suggest that the solution conditions used in Dole's work can lead to much greater aggregation and higher massto-charge ratio molecular ions than would be observed by conventional ESI/MS.

In summary, although ESI production of highly charged macromolecular ions is well established, this is the first study in which mass spectrometric data have confirmed the presence of macromolecular ions having low charge states. These species have not been detected previously because either the mass-to-charge ratio range was limited or there is discrimination (i.e., lower transmission efficiency) that is generally prevalent at higher mass-to-charge ratios for standard quadrupole mass filters. Any broadly applicable mechanism for ESI must take into account the possible formation of high mass-to-charge ratio (low charge state) molecular ions. We suggest that a variation of the charged-residue process appears most consistent with ESI/MS observations of macromolecules. Attempts to distinguish experimentally this mechanism from field evaporation of highly solvated molecular ions with mass spectrometric tools, however, will require a better understanding of the range of processes that contribute to the ions ultimately detected.

\section{Acknowledgments}

This work was supported by the Molecular Science Research Center of Pacific Northwest Laboratory through the U.S. Department of Energy. Pacific Northwest Laboratory is operated by Battelle Memorial Institute for the U.S. Department of Energy under Contract DE-AC06-76RLO 1830.

\section{References}

1. Fenn, J. B.; Mann, M.; Meng, C. K.; Wong, S. F.; Whitehouse, C. M. Mass Spectrom. Rev. 1990, 9, 37.

2. Smith, R. D.; Loo, J. A.; Ogorzalek Loo, R. R.; Busman, M.; Udseth, H. R. Mass Spectrom. Rev. 1991, 10, 359.

3. Iribarne, J. V.; Thomson, B. A. J. Chem. Phys. 1976, 64, 2287.

4. Thomson, B. A.; Iribarne, J. V. J. Chem. Phys, 1979, 71, 4451.

5. (a) Ikonomou, M. G.; Blades, A. T; Kebarle, P. Anal. Chem. 1990, 62, 957; (b) Ikonomou, M. G.; Blades, A. T.; Kebarle, P. Anal. Chem. 1991, 63, 1989.

6. Nohmi, T.; Fenn, J. B.; Shen, S. Proceedings of the 39th ASMS Conference on Mass Spectrometry and Allied Topics; Nashville, TN, May 19-24, 1991; p 443.

7. (a) Dole, M.; Mach, L. L.; Hunes, R. L.; Mobley, R. C.; Ferguson, L. P.; Alice, M. B. I. Chem. Phys. 1968, 49, 2240; (b) Mack, L. L.; Kralik, P; Rhuede, A.; Dole, M. I. Chem. Phys. $1970,52,4977$.

8. Röllgen, F. W.; Bramer-Weger, E.; Butfering, L. J. Prys. (Paris) $1987,48, \mathrm{C} 6$.

9. Schmelzeisen-Redeker, G.; Butfering, L.; Röllgen, F. W. Int. J. Mass Spectrom. Ion Processes 1989, 90, 139.

10. Raleigh, J. W. Philos. Mag. 1882, 14, 184. 
11. Nohmi, T.; Fenn, J. B. J. Am. Chem. Soc. 1992, 114, 3241.

12. (a) Ganem, B.; Li, Y.-T.; Henion, J. I. Am. Chem. Soc. 1991 113, 6294; (b) Ganem, B.; T.i, Y.-T.; Henion, J. J. Am. Chem. Soc. 1991, 113, 7818; (c) Katta, V; Chait, B. T. J. Am. Chem. Soc. 1991, 113, 8534; (d) Baca, M.; Kent, S. B. H. I. Am. Chem. Soc. 1992, 114, 3992; (e) Ganguly, A. K,; Pramarki, B. N.; Tsarbopoulos, A.; Covey, T. R.; Huang, E.; Fuhrman, S. A. I. Am Chem. Soc. 1992, 174, 6559; (f) Light-Wahl, K. J.; Springer, D. L.; Winger, B. E.; Edmonds, C. G.; Camp, D. G.; Thrall, B. D.; Smith, R. D. I. Am. Chem. Soc. 1993, 115, 803.

13. Smith, R. D.; Light-Wahl, K. J;; Winger, B. E.; Loo, J.A. Org. Mass Spectrom. 1992, 27, 811.

14. Chowdhury, S. K.; Katta, V,; Beavis, R. C.; Chait, B. T. J. Am. Soc. Mass Spectrom. 1990, 1, 382.

15. Winger, B. E.; Light-Wahl, K. J.; Udseth, H. R.; Smith, R. D., unpublished results.

16. Beuhler, R. J.; Friedman, L. J. J. Chem. Phys. 1982, 77, 2549.

17. Chowdhury, S. K.; Katta, V; Chait, B. T. Rapid Commun. Mass Spectrom. 1990, 4, 81.

18. (a) Ogorzalek Loo, R. R; Loo, J. A.; Udseth, H. R.; Fulton, J. L.; Smith, R. D. Rapid Commun. Mass Spectrom. 1992, 6, 159; (b) Ogorzalek Loo, R. R.; Udseth, H. R.; Smith, R. D. I. Am. Soc. Mass Spectrom. 1992, 3,695.

19. Smith, R. D.; Barinaga, C. J.; Udseth, H. R. Anal. Chem. 1988, $60,1948$.

20. Meng, C. K.; Fenn, J. B. Org. Mass Spectrom. 1991, 26, 542.

21. Loo, J. A.; Edmonds, C. G.; Udseth, H. R.; Smith, R. D. Anal. Chem. 1990, 62, 693.
22. Chowdhury, S. K.; Katta, V.; Chait, B. T. I. Am. Chem. Soc. 1990, 112, 9012.

23. Le Blanc, J. C. Y.; Betchemin, D.; Siu, K. W. M.; Guevremont, R.; Berman, S. S. Org. Mass Spectrom. 1991, 26, 831.

24. Volkin, D. B, Klibanov, A. M. In: Protein Function: A Practical Approach; Creighton, T. E., Ed.; IRL Press: Oxford, 1990; p 4.

25. Smith, R. D.; Loo, J. A.; Edmonds, C. G.; Barinaga, C. J.; Udseth, H. R. Anal. Chem. 1990, 62, 882.

26. (a) McLuckey, S. A.; Van Berkel, G. J.; Glish, G. L. J. Am. Chem. Soc. 1990, 112, 5668; (b) McLuckey, S. A.; Glish, G. L.; Van Berkel, G. J. Anal. Chem. 1991, 63, 1971.

27. Dayoff, M. D. Atlas of Protein Sequence and Structure, Vol. 5 , Suppl. 2; National Biomedical Research Foundation: Silver Spring, MD, 1976; $\mathrm{p} 97$.

28. Lüttgens, U.; Röllgen, F. W. Proceedings of the 39th ASMS Conference on Mass Spectrometry and Allied Topics; Nashville, TN, May 19-24, 1991; P 439.

29. Vestal, M. L.; Allen, M. H. Proceedings of the 39th ASMS Conference on Mass Spectrometry and Allied Topics; Nashville, TN, May 19-24, 1991; p 445.

30. Kelly, M. A.; Vestling, M. M.; Fenselau, C. C.; Smith, P. B. Org. Mass Spectrom. 1992, 27, 1143.

31. Winger, B. E.; Light-Wahl, K. J.; Smith, R. D. I. Am. Soc. Mass Spectrom. 1992, 3, 624.

32. Anacleto, J. F.; Pleasance, S.; Boyd, R. K. Org. Mass Spectrom. 1992, 27, 660.

33. Gieniec, I.; Mack, L. L.; Nakamae, K.; Gupta, C.; Kumar, V.; Dole, M. Biomed. Environ. Mass Spectrom. 1984, 11, 259. 